\title{
Gender difference in preference of specialty as a career choice among Japanese medical students
}

Ryuichi Kawamoto ${ }^{1,2^{*}}$, Daisuke Ninomiya ${ }^{1,2}$, Yoshihisa Kasai ${ }^{2}$, Tomo Kusunoki ${ }^{2}$, Nobuyuki Ohtsuka ${ }^{2}$, Teru Kumagi $i^{1}$ and Masanori Abe ${ }^{1}$

\begin{abstract}
Background: In Japan, the absolute deficiency of doctors and maldistribution of doctors by specialty is a significant problem in the Japanese health care system. The purpose of this study was to investigate the factors contributing to specialty preference in career choice among Japanese medical students.

Methods: A total of 368 medical students completed the survey giving an $88.2 \%$ response rate. The subjects comprised 141 women aged $21 \pm 3$ (range, 18-34) years and 227 men aged $22 \pm 4$ (range, 18-44) years. Binary Logistic regression analysis was performed using specialty preferences as the criterion variable and the factors in brackets as six motivational variables (e.g., Factor 1: educational experience; Factor 2: job security; Factor 3: advice from others; Factor 4: work-life balance; Factor 5: technical and research specialty; and Factor 6: personal reasons).

Results: Women significantly preferred pediatrics, obstetrics \& gynecology, and psychology than the men. Men significantly preferred surgery and orthopedics than the women. For both genders, a high odds ratio (OR) of "technical \& research specialty" and a low OR for "personal reasons" were associated with preference for surgery. "Technical \& research specialty" was positively associated with preference for special internal medicine and negatively for pediatrics. "Work-life balance" was positively associated with preference for psychology and negatively for emergency medicine. Among the women only, "technical \& research specialty" was negatively associated with preference for general medicine/family medicine and obstetrics \& gynecology, and "job security" was positively associated for general medicine/family medicine and negatively for psychology. Among men only, "educational experience" and "personal reasons" were positively, and "job security" was negatively associated with preference for pediatrics. For both genders, "work-life balance" was positively associated with preference for controllable lifestyle specialties.
\end{abstract}

Conclusion: We must acknowledge that Japanese medical students have dichotomized some motivations for their specialty preference based on gender. Systematic improvements in the working environment are necessary to solve these issues.

Keywords: Career choice, Gender difference, Japanese medical students

\footnotetext{
* Correspondence: rykawamo@m.ehime-u.ac.jp

${ }^{1}$ Department of Community Medicine, Ehime University Graduate School of Medicine, Toon-city, Ehime-ken 791-0295, Japan

${ }^{2}$ Department of Internal Medicine, Seiyo Municipal Nomura Hospital, 9-53

Nomura, Nomura-cho, Seiyo-city, Ehime-ken 797-1212, Japan
} 


\section{Background}

In Japan, there have been absolute deficiencies in the number of physicians. This number of doctors belongs to the lowest group in the Organization for Economic Cooperation and Development (OECD) [1]. In addition to the absolute deficiency of doctors, maldistribution of doctors among the various specialties is a serious problem in the Japanese health care system. A Japanese governmental report revealed that obstetrics \& gynecology, pediatrics, and anesthesiology have suffered a more severe shortage than other specialties [2]. Moreover, the decreasing tendency of doctors to choose internal medicine and surgery has accelerated [3].

In Japan, internal medicine showed the highest preference rate, followed by general surgery, pediatrics, and emergency medicine. The gender ratio varied according to the specialty [4], and the preference rates for general surgery, orthopedics, neurosurgery, and emergency medicine were significantly higher in men than in women, while those for obstetrics \& gynecology, pediatrics, and dermatology were significantly higher in women [5]. Women now account for about one third to half of medical students and have become a growing part of medical schools in Japan as well as the U.S. and Europe [6, 7], however female physicians are still underrepresented in some specialties such as surgery and emergency medicine [8].

Choosing a specialty is a complex process and may be influenced by several confounding factors. Distribution of medical students' career choices among specialties varies considerably. Recent studies in other countries identified several factors related to the choice and preference, such as gender, career opportunities, prestige, and income [9-11]. Enoch et al. demonstrated that specialties that feature a controllable lifestyle (control of work hours) were defined as anesthesiology, dermatology, emergency medicine, neurology, ophthalmology, otolaryngology, pathology, psychiatry, and radiology, while non-controllable lifestyle specialties were surgery, internal medicine, family practice, pediatrics, orthopedic surgery, and obstetrics \& gynecology [12]. In the USA, interest in surgery, pediatrics, and obstetrics \& gynecology has declined, but the popularity of controllable lifestyle fields such as radiology, psychiatry, dermatology, and ophthalmology has been increasing [13]. Controllable lifestyle is becoming an increasingly important factor in choice of specialty by medical students. Moreover, for surgical careers, the decision to have a family (e.g., children) was a more significant influence for women than men [14]. Thus, it is important to know the expectations of future physicians as they play a role in their career choice. There are many studies on career choice of medical students in Western countries. However, studies exploring the impact of gender differences regarding specialty preferences in career choice are still lacking in Japan.
The purpose of this study was to compare specialty preferences and the motivational factors of Japanese medical students. We investigated associations between motivational factors and specialty preference in order to determine the factors contributing to specialty preferences as a career.

\section{Methods}

\section{Participants}

We cross-sectionally conducted a survey of $1^{\text {st }}$ to $5^{\text {th }}$ year medical students $(N=450)$ from one Japanese regional university school of medicine. From April 2009 to 2013 (in Japan, the academic year begins in April), the selfadministered questionnaire was distributed and collected in class within the first 4 weeks of the start of medical school. Individual responses required a signature (anonymous responses were also permitted), and completion of the questionnaire was voluntary. The study was approved by the ethics committee of the Ehime University Graduate School of Medicine (IRB: 1507004), and informed consent was obtained from all subjects.

\section{Questionnaire}

We used a modified questionnaire enquiring about their specialty preference and to what extent their decision was influenced by a set of given criteria that were developed by Takeda et al. [15]. Questions on gender, age, academic year, admission from hometown, type of high school, admission by a special policy aimed directly at increasing the number of rural physicians as the main purpose (chiikiwaku in Japanese), student preparing for the entrance exam next year, presence of medical relatives, and growing up in a rural area were included.

Participants were asked to specify which of the following 14 medical specialties they intended to pursue: general medicine/family medicine (GM/FM), internal medicine, surgery, paediatrics, obstetrics \& gynaecology, psychiatry, anaesthesiology, emergency medicine, dermatology, orthopaedics, ophthalmology, otolaryngology, urology and radiology, or "other". They were instructed to choose their most preferred specialty and other specialties 'under consideration' with no restriction in the number. When "other" was chosen for a non-listed specialty, respondents were asked to specify which discipline they were choosing. In Japan, non-controllable lifestyle specialties are general medicine/family practice, special Internal medicine, surgery, pediatrics, obstetrics \& gynecology, anesthesiology, emergency medicine, and orthopedic surgery, and controllable lifestyle specialties are psychiatry, dermatology, ophthalmology, otolaryngology, urology, and radiology $[13,16]$. With regard to choice of a specialty, we [17] previously defined the following 6 factors based on the items that were grouped together: Factor 1: educational experience; Factor 2: job security; Factor 3: advice from others; Factor 4: work- 
life balance; Factor 5: technical and research specialty; and Factor 6: personal reasons. These 6 factors collectively accounted for $47.6 \%$ of the variance in responses. We calculated Cronbach's alpha coefficiencies which demonstrated internal consistency that ranged between 0.55 and 0.84 . The subscale response to the influences were rated on a 4point likert scale $(1=$ not at all, $2=$ not particularly, $3=$ fairly well, and $4=$ extremely well).

\section{Data analysis}

Statistical analysis was performed using IBM SPSS Statistics Version 21 (Statistical Package for Social Science, Chicago, IL, USA). Data are presented as the mean \pm standard deviation (SD) unless otherwise specified. Gender differences in the subscales by level of interest in choice of a specialty as a career were examined using Mann-Whitney $U$ test for continuing variables or $\chi^{2}$-test for categorical variables. Finally, Binary Logistic regression analysis was performed in order to derive confounding factors associated with level of interest in choosing a specialty as a career by gender. The interactive effect of gender and confounding factors was evaluated using a general linear model. A $p$-value $<0.05$ was considered significant.

\section{Results}

\section{Characteristics of female and male students}

Of 417 students from whom the questionnaire could be collected, 368 completed the survey giving an $88.2 \%$ response rate. The subjects comprised 141 women aged $21 \pm$ 3 (range, 18-34) years and 227 men aged $22 \pm 4$ (range, 18-44) years (Table 1). "Student preparing for the entrance exam next year" ranked higher in men than in women and "having a role model" ranked higher in women than in men. There were no inter-group differences regarding age, academic year, admission from home, public high school graduation, combined junior high and high school graduation, admission by a special policy, presence of medical relatives, and growing up in a rural area.

\section{Female and male students' career preferences}

Table 2 shows the levels of interest for specialty choices as a career by female and male students. The most common specialties chosen by the students were general medicine/ family medicine (18.8 \%), surgery (9.5\%), pediatrics (7.6 \%), special Internal medicine $(7.1 \%)$, emergency medicine (4.6\%), orthopedics (3.8\%), and urology (3.3\%). The other specialties chosen are listed in Table 2 . Women significantly preferred pediatrics, obstetrics \& gynecology, and psychology than men. Men significantly preferred surgery and orthopedics than women.
Table 1 Characteristics of female and male medical students

\begin{tabular}{|c|c|c|c|c|}
\hline & Total & Women & Men & $P$-value \\
\hline Characteristics & $N=368$ & $N=141$ & $N=227$ & \\
\hline Age, years & $21 \pm 4$ & $21 \pm 3$ & $22 \pm 4$ & 0.258 \\
\hline \multicolumn{5}{|l|}{ Academic year } \\
\hline $1^{\text {st }}-2^{\text {nd }}$ & $179(48.6)$ & $68(48.2)$ & $111(48.9)$ & \multirow[t]{2}{*}{0.915} \\
\hline $3^{\text {rd }}-5^{\text {th }}$ & $189(51.4)$ & $73(51.8)$ & $116(51.1)$ & \\
\hline \multicolumn{5}{|c|}{ Admission from hometown } \\
\hline Yes & $168(45.7)$ & $64(45.4)$ & $104(45.8)$ & \multirow[t]{2}{*}{1.000} \\
\hline No & $200(54.3)$ & $77(54.6)$ & $123(54.2)$ & \\
\hline \multicolumn{5}{|c|}{ Public high school graduation } \\
\hline Yes & $195(53.0)$ & $76(53.9)$ & $119(52.4)$ & \multirow[t]{2}{*}{0.830} \\
\hline No & $173(47.0)$ & $65(46.1)$ & $108(47.6)$ & \\
\hline
\end{tabular}

Combined junior high and high school graduation

$\begin{array}{lllll}\text { Yes } & 164(44.6) & 64(45.4) & 100(44.1) & 0.830 \\ \text { No } & 204(55.4) & 77(54.6) & 127(55.9) & \end{array}$

$\begin{array}{llll}\text { No } & 204(55.4) & 77(54.6) & 127(55.9)\end{array}$

Admission by a special policy

$\begin{array}{lllll}\text { Yes } & 23(6.3) & 6(4.3) & 17(7.5) & 0.270 \\ \text { No } & 345(93.8) & 135(95.7) & 210(92.5) & \end{array}$

Student preparing for the entrance exam next year

\begin{tabular}{|c|c|c|c|c|}
\hline Yes & $175(47.6)$ & $55(39.0)$ & $120(52.9)$ & 0.010 \\
\hline No & $193(52.4)$ & $86(61.0)$ & $107(47.1)$ & \\
\hline \multicolumn{5}{|c|}{ Presence of medical relatives } \\
\hline Yes & $162(44.0)$ & $63(44.7)$ & 99 (43.6) & \multirow[t]{2}{*}{0.914} \\
\hline No & $206(56.0)$ & $78(55.3)$ & $128(56.4)$ & \\
\hline \multicolumn{5}{|c|}{ Growing up in a rural area } \\
\hline Yes & $47(12.8)$ & $19(13.5)$ & $28(12.3)$ & \multirow[t]{2}{*}{0.750} \\
\hline No & $321(87.2)$ & $122(86.5)$ & $199(87.7)$ & \\
\hline \multicolumn{5}{|c|}{ Presence of a role model } \\
\hline Yes & $152(41.3)$ & $67(47.5)$ & $85(37.4)$ & \multirow[t]{2}{*}{0.064} \\
\hline No & $216(58.7)$ & $74(52.5)$ & $142(62.6)$ & \\
\hline
\end{tabular}

Data are presented as number (\%) or mean \pm standard deviation. $P$-value from Mann-Whitney U-test for continuing variables and $\mathrm{x}^{2}$-test for categorical variables. Bold numbers indicate significance $(p<0.05)$

Rating of motivational factors for specialty preference Table 3 shows female and male motivational factors for specialty preference. Compared with men, women considered "memorable experience in a class or clinical rotation", "encounter with role model teachers", "advice from teachers/consultants", and "interest in targeted populations such as children or the elderly" to be more important reasons for choosing a specialty, and "expected income" to be less important.

\section{Motivational factors associated with specialty preference}

Table 4 shows the similarities and differences in both genders regarding motivational factors and specialty preference after adjusting other characteristics. For both genders, a high odds ratio (OR) for the "technical \& research 
Table 2 Female and male students' career preferences

\begin{tabular}{|c|c|c|c|c|c|}
\hline \multirow[b]{2}{*}{ Specialty preference } & \multirow[b]{2}{*}{ Choice } & \multirow{2}{*}{$\begin{array}{l}\text { Total } \\
N=368\end{array}$} & \multirow{2}{*}{$\begin{array}{l}\text { Women } \\
N=141\end{array}$} & \multirow{2}{*}{$\begin{array}{l}\text { Men } \\
N=227\end{array}$} & \multirow[t]{2}{*}{$P$-value } \\
\hline & & & & & \\
\hline \multirow[t]{2}{*}{ General medicine/Family medicine } & First & $69(18.8)$ & $27(19.1)$ & $42(18.5)$ & 0.695 \\
\hline & Second & $156(42.4)$ & $63(44.7)$ & $93(41.0)$ & \\
\hline \multirow[t]{2}{*}{ Special Internal medicine } & First & $26(7.1)$ & $6(4.3)$ & $20(8.8)$ & 0.172 \\
\hline & Second & $82(22.3)$ & $29(20.6)$ & $53(23.3)$ & \\
\hline \multirow[t]{2}{*}{ Surgery } & First & $35(9.5)$ & $6(4.3)$ & $29(12.8)$ & $<0.001$ \\
\hline & Second & $113(30.7)$ & $33(23.4)$ & $80(35.2)$ & \\
\hline \multirow[t]{2}{*}{ Pediatrics } & First & $28(7.6)$ & $12(8.5)$ & $16(7.0)$ & 0.038 \\
\hline & Second & $102(27.7)$ & $49(34.8)$ & $53(23.3)$ & \\
\hline \multirow[t]{2}{*}{ Obstetrics \& Gynecology } & First & $11(3.0)$ & $10(7.1)$ & $1(0.4)$ & $<0.001$ \\
\hline & Second & $44(12.0)$ & $28(19.9)$ & $16(7.0)$ & \\
\hline \multirow[t]{2}{*}{ Emergency medicine } & First & $17(4.6)$ & $4(2.8)$ & $13(5.7)$ & 0.436 \\
\hline & Second & $96(26.1)$ & $37(26.2)$ & $59(26.0)$ & \\
\hline \multirow[t]{2}{*}{ Psychology } & First & $8(2.2)$ & $4(2.8)$ & $4(1.8)$ & 0.038 \\
\hline & Second & $48(13.0)$ & $26(18.4)$ & $22(9.7)$ & \\
\hline \multirow[t]{2}{*}{ Anesthesiology } & First & $6(1.6)$ & $4(2.8)$ & $2(0.9)$ & 0.351 \\
\hline & Second & $46(12.5)$ & $17(12.1)$ & $29(12.8)$ & \\
\hline \multirow[t]{2}{*}{ Orthopedics } & First & $14(3.8)$ & 0 & $14(6.2)$ & $<0.001$ \\
\hline & Second & 69 (18.8) & $10(7.1)$ & $59(26.0)$ & \\
\hline \multirow[t]{2}{*}{ Dermatology } & First & $3(0.8)$ & $2(1.4)$ & $1(0.4)$ & 0.112 \\
\hline & Second & $32(8.7)$ & $17(12.1)$ & $15(6.6)$ & \\
\hline \multirow[t]{2}{*}{ Ophthalmology } & First & $6(1.6)$ & $3(2.1)$ & $3(1.3)$ & 0.531 \\
\hline & Second & $30(8.2)$ & $9(6.4)$ & $21(9.3)$ & \\
\hline \multirow[t]{2}{*}{ Otolaryngology } & First & $4(1.1)$ & $0(0)$ & $4(1.8)$ & 0.196 \\
\hline & Second & $26(7.1)$ & $8(5.7)$ & $18(7.9)$ & \\
\hline \multirow[t]{2}{*}{ Urology } & First & $12(3.3)$ & $3(2.1)$ & $9(4.0)$ & 0.385 \\
\hline & Second & 0 & 0 & 0 & \\
\hline \multirow[t]{2}{*}{ Radiology } & First & $5(1.4)$ & $4(2.8)$ & $1(0.4)$ & 0.053 \\
\hline & Second & $23(6.3)$ & $12(8.5)$ & $11(4.8)$ & \\
\hline \multirow[t]{2}{*}{ Others } & First & $1(0.3)$ & $1(0.7)$ & 0 & 0.350 \\
\hline & Second & $8(2.2)$ & $4(2.8)$ & $4(1.8)$ & \\
\hline
\end{tabular}

Data are presented as numbers (\%). $P$-value from $x^{2}$-test. Bold numbers indicate significance $(p<0.05)$

specialty", but a low OR for "personal reasons" were associated with preference for surgery. "Technical \& research specialty" was positively associated with preference for special internal medicine and negatively for pediatrics. "Worklife balance" was positively associated with preference for psychology and negatively for emergency medicine. It was only among women that "technical \& research specialty" was negatively associated with preference for general medicine/family medicine and obstetrics \& gynecology, and "job security" was positively associated with general medicine/family medicine, but negatively with psychology. It was only among men that "educational experience" and "personal reasons" were positively associated, but "job security negatively associated with preference for pediatrics. When gender as an interaction term was studied in the association between motivational factors and preference of specialty, two significant differences were found. Each low ORs of "technical \& research specialty" for general medicine/ family medicine and "educational experience" for anesthesiology had a stronger association with female than male preference.

\section{Motivational factors associated with controllable and} non-controllable lifestyle groups of specialties by gender For both genders, "work-life balance" was positively associated with preference for controllable lifestyle specialties (Table 5). Among women only, a high OR of "advice 
Table 3 Rating of female and male motivational factors for specialty preference

\begin{tabular}{|c|c|c|c|c|}
\hline & Total & Women & Men & \\
\hline & $N=368$ & $N=141$ & $N=227$ & P-value \\
\hline Factor 1: Educational experience & $3.0 \pm 0.6$ & $3.1 \pm 0.7$ & $3.0 \pm 0.6$ & 0.009 \\
\hline 15) Received excellent teachings & $3.1 \pm 0.7$ & $3.2 \pm 0.7$ & $3.1 \pm 0.7$ & 0.111 \\
\hline 14) Memorable experience in a class or clinical rotation & $3.0 \pm 0.7$ & $3.2 \pm 0.8$ & $2.9 \pm 0.7$ & 0.005 \\
\hline 16) Comfortable atmosphere at the specialty department & $3.1 \pm 0.8$ & $3.2 \pm 0.8$ & $3.1 \pm 0.7$ & 0.268 \\
\hline 17) Encounter with role model teachers & $2.9 \pm 0.9$ & $3.1 \pm 0.9$ & $2.8 \pm 0.9$ & 0.024 \\
\hline Factor 2: Job security & $2.1 \pm 0.6$ & $2.1 \pm 0.5$ & $2.1 \pm 0.6$ & 0.392 \\
\hline 19) Advice/Expectation of parents & $2.2 \pm 0.9$ & $2.3 \pm 0.9$ & $2.2 \pm 0.9$ & 0.178 \\
\hline 26) Expected income & $2.3 \pm 0.8$ & $2.1 \pm 0.7$ & $2.4 \pm 0.9$ & 0.002 \\
\hline 24) Ease of opening practice & $2.0 \pm 0.8$ & $1.9 \pm 0.7$ & $2.1 \pm 0.8$ & 0.077 \\
\hline 25) Expectation to inherit practice of my parents/relatives & $1.6 \pm 0.8$ & $1.5 \pm 0.7$ & $1.7 \pm 0.9$ & 0.121 \\
\hline 23) Job availability & $2.5 \pm 0.8$ & $2.6 \pm 0.8$ & $2.5 \pm 0.9$ & 0.250 \\
\hline Factor 3: Advice from others & $2.1 \pm 0.7$ & $2.1 \pm 0.8$ & $2.1 \pm 0.7$ & 0.175 \\
\hline 20) Advice from senior students/residents & $2.1 \pm 0.9$ & $2.2 \pm 0.9$ & $2.0 \pm 0.8$ & 0.154 \\
\hline 21) Advice from teachers/consultants & $2.2 \pm 0.9$ & $2.4 \pm 1.0$ & $2.2 \pm 0.9$ & 0.037 \\
\hline 22) Influence of friends & $2.0 \pm 0.8$ & $1.9 \pm 0.7$ & $2.0 \pm 0.8$ & 0.578 \\
\hline Factor 4: Work-life balance & $2.4 \pm 0.7$ & $2.5 \pm 0.7$ & $2.4 \pm 0.7$ & 0.087 \\
\hline 27) Working hours & $2.4 \pm 0.9$ & $2.5 \pm 0.9$ & $2.3 \pm 0.9$ & 0.080 \\
\hline 28) Attainable lifestyle & $2.7 \pm 0.9$ & $2.8 \pm 0.9$ & $2.7 \pm 0.9$ & 0.106 \\
\hline 30) Risk of my malpractice law suits & $2.2 \pm 0.8$ & $2.2 \pm 0.8$ & $2.2 \pm 0.8$ & 0.587 \\
\hline Factor 5: Technical \& research specialty & $3.0 \pm 0.6$ & $3.0 \pm 0.6$ & $3.0 \pm 0.6$ & 0.766 \\
\hline 5) Interest in the surgical procedures or technologies & $3.1 \pm 0.8$ & $3.0 \pm 0.9$ & $3.1 \pm 0.8$ & 0.338 \\
\hline 6) Mastering the specialty & $3.0 \pm 0.8$ & $2.9 \pm 0.8$ & $3.0 \pm 0.7$ & 0.298 \\
\hline 4) Interest in the research or scientific aspects & $3.0 \pm 0.8$ & $3.0 \pm 0.8$ & $3.0 \pm 0.8$ & 0.884 \\
\hline 2) Interest in the organ specialty & $3.0 \pm 0.8$ & $3.0 \pm 0.8$ & $3.0 \pm 0.8$ & 0.377 \\
\hline Factor 6: Personal reasons & $2.5 \pm 0.6$ & $2.6 \pm 0.6$ & $2.5 \pm 0.7$ & 0.290 \\
\hline 11) I suffering(ed) from an illness of the specialty & $2.2 \pm 1.0$ & $2.2 \pm 1.0$ & $2.3 \pm 1.1$ & 0.520 \\
\hline 12) Friend/family suffers(ed) from an illness of the specialty & $2.2 \pm 1.0$ & $2.5 \pm 1.0$ & $2.4 \pm 1.1$ & 0.401 \\
\hline 13) Became interested in the specialty before medical school & $2.5 \pm 0.9$ & $2.6 \pm 0.9$ & $2.5 \pm 0.9$ & 0.124 \\
\hline 3) Interest in the targeted populations such as children or the elderly & $2.9 \pm 0.8$ & $3.0 \pm 0.8$ & $2.8 \pm 0.8$ & 0.010 \\
\hline
\end{tabular}

Data are presented as mean \pm standard deviation. $P$-value from Mann-Whitney $\mathrm{U}$ test. Bold numbers indicate significance $(p<0.05)$

from others", but a low OR of "educational experience" was associated with preference for controllable lifestyle specialties.

\section{Discussion}

The purpose of this study was to investigate and compare male and female preferences for specialties and motivational factors among Japanese medical students. Our results showed some gender differences in choice of specialty by the medical students. Women considered "interest in targeted populations such as children or the elderly" to be a more important factor for choosing a specialty, and "expected income" to be less important. Moreover, they significantly preferred pediatrics, obstetrics \& gynecology, and psychology than men. Men significantly preferred surgery and orthopedics. There were some differences between the genders regarding motivational factors associated with choice of specialty. Lastly, both the male and female students who preferred the controllable lifestyle specialties considered work-life balance to be important.

Our findings provide some important insights in medical student's choice of specialty. In Japan, Fukuda et al. [5] reported that internal medicine has the highest preference rate followed by general surgery, pediatrics, and emergency medicine, and the preference rates for general surgery, orthopedics, neurosurgery, and emergency medicine were significantly higher in men than in women, while those for obstetrics \& gynecology, pediatrics, and dermatology were higher in women. Gender differences in 
Table 4 Motivational factors associated with specialty preference by gender

\begin{tabular}{|c|c|c|c|}
\hline \multirow[b]{3}{*}{ Specialty preference } & \multicolumn{2}{|c|}{ Odds ratio $(95 \% \mathrm{Cl})$} & \multirow{3}{*}{$\begin{array}{l}\text { Gender interaction } \\
p \text {-value }\end{array}$} \\
\hline & \multirow{2}{*}{$\begin{array}{l}\text { Women } \\
N=141\end{array}$} & Men & \\
\hline & & $N=227$ & \\
\hline \multicolumn{4}{|c|}{ General medicine/Family medicine (Yes/No) } \\
\hline Job security & \multicolumn{2}{|l|}{$2.21(1.04-4.69)$} & 0.104 \\
\hline Technical \& research specialty & \multicolumn{2}{|l|}{$0.29(0.14-0.59)$} & 0.022 \\
\hline \multicolumn{4}{|l|}{ Special Internal medicine (Yes/No) } \\
\hline Technical \& research specialty & $2.01(1.10-3.68)$ & $2.28(1.32-3.94)$ & 0.737 \\
\hline \multicolumn{4}{|l|}{ Surgery (Yes/No) } \\
\hline Work-life balance & $0.54(0.28-1.06)$ & -—— & 0.373 \\
\hline Technical \& research specialty & $3.41(1.39-8.35)$ & $2.94(1.70-5.10)$ & 0.732 \\
\hline Personal reasons & $0.18(0.07-0.44)$ & $0.49(0.31-0.79)$ & 0.276 \\
\hline \multicolumn{4}{|l|}{ Pediatrics (Yes/No) } \\
\hline Educational experience & -—- & $1.94(1.16-3.25)$ & 0.060 \\
\hline Job security & -—- & $0.52(0.31-0.85)$ & 0.345 \\
\hline Technical \& research specialty & $0.44(0.24-0.82)$ & $0.55(0.32-0.93)$ & 0.616 \\
\hline Personal reasons & -——- & $1.66(1.01-2.73)$ & 0.207 \\
\hline \multicolumn{4}{|l|}{ Obstetrics \& Gynecology (Yes/No) } \\
\hline Educational experience & $0.56(0.29-1.07)$ & - - - & 0.154 \\
\hline Advice from others & $1.69(0.91-3.14)$ & --— & 0.175 \\
\hline Technical \& research specialty & $0.44(0.22-0.89)$ & -—- & 0.512 \\
\hline \multicolumn{4}{|l|}{ Psychology (Yes/No) } \\
\hline Job security & $0.19(0.04-0.80)$ & -—- & 0.135 \\
\hline Advice from others & $2.45(1.10-5.46)$ & - - - & 0.067 \\
\hline Work-life balance & $3.01(1.22-7.43)$ & $1.88(1.02-3.49)$ & 0.537 \\
\hline Personal reasons & $2.06(0.93-4.56)$ & -—- & 0.571 \\
\hline \multicolumn{4}{|l|}{ Anesthesiology (Yes/No) } \\
\hline Educational experience & $0.32(0.15-0.67)$ & - - - & 0.032 \\
\hline Technical \& research specialty & -—— & $1.81(0.91-3.62)$ & 0.412 \\
\hline \multicolumn{4}{|l|}{ Emergency medicine (Yes/No) } \\
\hline Job security & -—— & $0.55(0.29-1.02)$ & 0.070 \\
\hline Advice from others & -—— & $1.83(1.09-3.09)$ & 0.152 \\
\hline Work-life balance & $0.46(0.23-0.90)$ & $0.53(0.32-0.89)$ & 0.410 \\
\hline Technical \& research specialty & $2.19(0.98-4.90)$ & -—- & 0.090 \\
\hline Personal reasons & $0.45(0.21-0.97)$ & $0.61(0.38-0.98)$ & 0.903 \\
\hline \multicolumn{4}{|l|}{ Orthopedics (Yes/No) } \\
\hline Educational experience & $0.21(0.08-0.57)$ & -—- & 0.122 \\
\hline Job security & -—— & $2.17(0.96-4.88)$ & 0.885 \\
\hline Advice from others & $2.30(0.85-6.25)$ & -—-- & 0.070 \\
\hline Work-life balance & $4.87(1.63-14.6)$ & - - - & 0.227 \\
\hline
\end{tabular}

Yes, first and second choice. Data were adjusted for all confounding factors in Tables 1 and 3 by Binary Logistic regression analysis, and presented regarding motivational factors as the final model. Bold numbers indicate significance $(p<0.05)$

Cl confidence interval

choice and preference of specialty among medical students are common internationally, and previous investigations in other countries also demonstrated that pediatrics and obstetrics \& gynecology are predominantly preferred by women, surgery by men, and internal medicine is pursued by both genders $[14,18,19]$. Similar patterns were 
Table 5 Motivational factors associated with controllable and non-controllable lifestyle groups of specialties by gender

\begin{tabular}{|c|c|c|c|}
\hline \multirow[b]{3}{*}{ Specialty preference } & \multicolumn{2}{|c|}{ Odds ratio $(95 \% \mathrm{Cl})$} & \multirow{3}{*}{$\begin{array}{l}\text { Gender Interaction } \\
p \text {-value }\end{array}$} \\
\hline & \multirow{2}{*}{$\begin{array}{l}\text { Women } \\
N=141\end{array}$} & Men & \\
\hline & & $N=227$ & \\
\hline \multicolumn{4}{|c|}{ Non-controllable lifestyle group (Yes/No) } \\
\hline Technical \& research specialty & \multicolumn{2}{|l|}{$0.16(0.02-1.53)$} & 0.156 \\
\hline Personal reasons & \multicolumn{2}{|l|}{$0.15(0.02-1.35)$} & 0.783 \\
\hline \multicolumn{4}{|l|}{ Controllable lifestyle group (Yes/No) } \\
\hline Educational experience & $0.45(0.23-0.88)$ & $0.67(0.42-1.06)$ & 0.683 \\
\hline Job security & $0.36(0.13-1.01)$ & -一- - & 0.104 \\
\hline Advice from others & $2.24(1.11-4.54)$ & -——- & 0.105 \\
\hline Work-life balance & $4.27(1.94-9.40)$ & $1.84(1.24-2.73)$ & 0.056 \\
\hline Technical \& research specialty & $0.56(0.29-1.09)$ & -—- & 0.099 \\
\hline
\end{tabular}

Non-controllable lifestyle group of specialties consisted of general medicine/family practice, special Internal medicine, surgery, pediatrics, obstetrics \& gynecology, anesthesiology, emergency medicine, and orthopedics, and controllable lifestyle were defined as psychiatry, dermatology, ophthalmology, otolaryngology, urology, and radiology. Yes, first and second choice. Data were adjusted for all confounding factors in Tables 1 and 3 by Binary Logistic regression analysis, and presented regarding motivational factors as the final model. Bold numbers indicate significance $(p<0.05)$

$\mathrm{Cl}$ confidence interval

observed in our study, and gender-related specialty preferences were apparent. The fact that more women than men preferred obstetrics \& gynecology and pediatrics could be explained by the high number of female physicians who are in these specialties and this has been the trend over the past decades. Hence, there are more same gender role models [20]. In Sweden, however, gender similarities among medical students regarding specialty preference are striking and contrast with research from other western countries [20]. Among Saudi undergraduate medical students, most women preferred surgery, followed by pediatrics and ophthalmology [19]. Individual preferences change extensively over time. Workload in these specialties is expected to increase as the number of female doctors increase due to changes in life circumstances such as pregnancies and child-rearing.

Women and men differed somewhat regarding the importance placed on the six motivational factors. Women rated "educational experience" (e.g., "memorable experience in a class" or "clinical rotation and "encounter with role model teachers"), "advice from teachers/consultants", and "interest in targeted populations such as children or the elderly" higher, and "expected income" lower than men. Women tended to place a higher value on comprehensive patient care and existence of role models than men in choice of specialty. This is consistent with past work $[10,21,22]$ indicating that women are more relationshiporiented than men.

Aasland et al. [23] showed that medical students who prefer surgery or internal medicine were more motivated by medical challenges and career possibilities, while those who prefer psychiatry or general medicine are more motivated by conditions such as variety and time for own family. Prestige of a specialty was less emphasized after graduation while the optimal combination of an interesting job and a good private life increased [23].

For both genders, work-life balance was an important factor that contributed to students preferring specialties in the controllable life style group. Controllable lifestyle was found to be strongly associated with recent trends in choice of specialty for both genders, and could not be explained solely by specialty preferences of women [24]. Dorsey et al. showed that the percentage of women choosing specialties with a controllable lifestyle increased from $18 \%$ in 1996 to $36 \%$ in 2003 and the percentage for men grew from 28 to $45 \%$ [24]. Students for whom work-life balance was extremely important (odds ratio $[\mathrm{OR}]=0.6$ ) were less likely to prefer surgery. Students for whom work-life balance (OR = $2.2)$ and continuity of care $(\mathrm{OR}=2.1)$ were extremely important were more likely to prefer general practice [25]. Understanding that perceptions towards prestige and lifestyle differ among students may be useful when advising medical students about choice of specialty [26].

There are some limitations to this study. First, our crosssectional study design does not eliminate potential causal relationships between the motivational factors of medical students and career choice. Second, this study involved a limited number of students who belong to one local university. Therefore, the demographics and referral source may limit generalizability. Third, as we used a self-administered questionnaire developed for 1 st to 5 th year medical students, some of the characteristics (such as motivational factors and choice of specialty) examined appeared to be more suitable for students in a higher academic year. Fourth, our study measured students' interest in a career, but not their actual choice after graduation. Future research using longitudinal data collection will enable us to monitor the relationship between early stated interest and actual behavior. 


\section{Conclusion}

The present study showed that medical students have a positive and realistic picture of what choosing a specialty as a career involves. Women and men differed somewhat in the importance they put on about six motivational factors. We must acknowledge that Japanese medical students have dichotomized some motivations for their preference of specialty and that is based on gender. Gender differences and mismatch of choices influence the current and future maldistributin of specialties. In Japan, systematic improvements in the work environment are necessary to solve these issues.

\section{Acknowledgments}

None.

\section{Funding}

This work was supported by Grant-in-Aid for Scientific Research (C) (20152017). No additional external funding was received for this study. The funders had no role in the study design, data collection and analysis, decision to publish, or preparation of the manuscript.

\section{Availability of data and materials}

The participant survey data supporting the conclusions of this manuscript are not publicly available due to protection of the privacy of the participants.

\section{Authors' contributions}

RK and MA participated in the design of the study, performed the statistical analysis and drafted the manuscript. RK, DN, YK, ToK, NO, and TeK contributed to the acquisition and interpretation of data. RK, DN, NO, and TeK contributed to the conception and design of the statistical analysis. RK and TeK conceived of the study, participated in its design, coordination and helped to draft the manuscript. All authors read and approved the manuscript.

\section{Competing interests}

The authors declare that they have no competing interests.

\section{Consent for publication}

Not applicable.

\section{Ethics approval and consent to participate}

The study was approved by the ethics committee of Ehime University Graduate School of Medicine (No. 15007004) and informed consent was obtained from all subjects participating in the study.

Received: 11 May 2016 Accepted: 1 November 2016

Published online: 10 November 2016

\section{References}

1. Organization for Economic Cooperation and Development. OECD health data 2014 (report). OECD. Doctors consultations, number per capita. (Online) 2015 Available: http://www.oecd.org/health/healthdata (Accessed 7 Apr 2014)

2. Yoshida A. Physicians' career building and their shortage in some specialties. Japan Labor Rev. 2011:8:5-21.

3. Koike S, Ide H, Yasunaga H, Kodama T, Matsumoto S, Imamura T. Postgraduate training and career choices: an analysis of the National Physicians Survey in Japan. Med Educ. 2010;44:289-97.

4. Kawakami Y. Support system for women doctors. JMAJ. 2011;54:136-8.

5. Fukuda Y, Harada T. Gender differences in specialty preference and mismatch with real needs in Japanese medical students. BMC Med Educ. 2010;10:15.

6. Boulis AK, Jacobs JA. The changing face of medicine: women doctors and the evolution of health care in America Ithaca, N.Y. London: Cornell University ILR Press; 2008.

7. Riska E. Gender and medical career. Maturitas. 2011:68:264-7.

8. Goldacre MJ, Goldacre R, Lambert TW. Doctors who considered but did not pursue specific clinical specialties as careers: questionnaire surveys. J R Soc Med. 2012;105:166-76
9. Puertas EB, Arósquipa C, Gutiérrez D. Factors that influence a career choice in primary care among medical students from high-, middle-, and low-income countries: a systematic review. Rev Panam Salud Publica. 2013;34:351-8.

10. Lefevre JH, Roupret M, Kerneis S, Karila L. Career choices of medical students: a national survey of 1780 students. Med Educ. 2010;44:603-12.

11. Deutsch T, Hönigschmid P, Frese T, Sandholzer H. Early community-based family practice elective positively influences medical students' career considerations-a pre-post-comparison. BMC Fam Pract. 2013;14:24.

12. Enoch L, Chibnall JT, Schindler DL, Slavin SJ. Association of medical student burnout with residency specialty choice. Med Educ. 2013;47:173-81.

13. DeZee KJ, Byars LA, Magee CD, Rickards G, Durning SJ, Maurer D. The R.O.A D. confirmed: ratings of specialties' lifestyles by fourth-year US medical students with a military service obligation. Fam Med. 2013:45:240-6.

14. Alers M, van Leerdam L, Dielissen P, Lagro-Janssen A. Gendered specialities during medical education: a literature review. Perspect Med Educ. 2014:3:163-78.

15. Takeda Y, Morio K, Snell L, Otaki J, Takahashi M, Kai I. Characteristic profiles among students and junior doctors with specific career preferences. BMC Med Educ. 2013;13:125.

16. Dorsey ER, Jarjoura D, Rutecki GW. Influence of controllable lifestyle on recent trends in specialty choice by US medical students. JAMA. 2003;290:1173-8.

17. Kawamoto R, Ninomiya D, Kasai Y, Kusunoki T, Ohtsuka N, Kumagi T, Abe M. Factors associated with the choice of general medicine as a career among Japanese medical students. Med Educ Online. 2016;21:29448.

18. van Tongeren-Alers $\mathrm{M}$, van Esch $\mathrm{M}$, Verdonk $\mathrm{P}$, Johansson $\mathrm{E}$, Hamberg $\mathrm{K}$, Lagro-Janssen T. Are new medical students' specialty preferences gendered? Related motivational factors at a Dutch medical school. Teach Learn Med. 2011;23:263-8.

19. Mehmood SI, Kumar A, Al-Binali A, Borleffs JC. Specialty preferences: trends and perceptions among Saudi undergraduate medical students. Med Teach. 2012;34:S51-60.

20. Diderichsen S, Johansson EE, Verdonk P, Lagro-Janssen T, Hamberg K. Few gender differences in specialty preferences and motivational factors: a cross-sectional Swedish study on last-year medical students. BMC Med Educ. 2013;13:39

21. Neittaanmäki L, Luhtala R, Virjo I, Kumpusalo $E$, Mattila $K$, Jääskeläinen $M$, Kujala S, Isokoski M. More women enter medicine: young doctors' family origin and career choice. Med Educ. 1993:27:440-5.

22. Heiligers PJ. Gender differences in medical students' motives and career choice. BMC Med Educ. 2012;12:82.

23. Aasland OG, Røvik JO, Wiers-Jenssen J. Motives for choice of specialty during and after medical school. Tidsskr Nor Laegeforen. 2008;128:1833-7.

24. Dorsey ER, Jarjoura D, Rutecki GW. The influence of controllable lifestyle and sex on the specialty choices of graduating U.S. medical students, 1996-2003. Acad Med. 2005:80:791-6.

25. Cleland J, Johnston PW, French FH, Needham G. Associations between medical school and career preferences in Year 1 medical students in Scotland. Med Educ 2012:46:473-84.

26. Lee CW. Gender difference and specialty preference in medical career choice Korean J Med Educ. 2013;25:15-21.
Submit your next manuscript to BioMed Central and we will help you at every step:

- We accept pre-submission inquiries

- Our selector tool helps you to find the most relevant journal

- We provide round the clock customer support

- Convenient online submission

- Thorough peer review

- Inclusion in PubMed and all major indexing services

- Maximum visibility for your research

Submit your manuscript at www.biomedcentral.com/submit
) Biomed Central 